\title{
INFLUENCE OF INTERFACIAL EFFECTS ON X-RAY DIFFRACTION SPECTRA OF THE $[\mathrm{GaAs}]_{n}[\mathrm{AlAs}]_{m}$ SUPERLATTICES* $^{*}$
}

\begin{abstract}
Grzegorz Gladyszewski
Institute of Physics, MCS University, pl. M.Curie-Skłodowskiej 1, 20-031 Lublin, Poland

(Received August 8, 1990)

Influence of interdiffusion, terrace growth, and macroscopic uniformity on $\mathrm{X}$-ray diffraction spectra of $[\mathrm{GaAs}]_{n}[\mathrm{AlAs}]_{m}$ superlattices are presented. High-angle spectra are analysed on the basis of the kinematic theory of diffraction, whereas low-angle spectra are analysed using the dynamic theory. Computer-simulated (using a Monte-Carlo method) spectra based on a "statistical" model of the superlattice structure are applied in the analysis.
\end{abstract}

PACS numbers: 61.10.Dp, 68.65.+g

$\mathrm{X}$-ray diffraction measurements are frequently used to examine structural parameters of semiconductor superlattices - mainly because the X-ray differaction spectra are very sensitive to interfacial effects [1]. These measurements are nondestructive and relatively simple but precise analysis of the spectra is difficult because we obtain a complicated image resulting from the influence of different structural parameters: a verage numbers of monolayers in the superlattice unit cell, fluctuations of these values, thickness of interdiffused region at the interface, the macroscopic uniformity of the multilayer across the film, etc [2-4].

For the precise quantitative description of the interfacial effects high- and low-angle X-ray diffraction spectra should be examined. The high-angle spectra can be interpreted on the basis of the kinematic theory of diffraction, whereas the low-angle spectra should be analysed using the dynamic theory. The $[\mathrm{GaAs}]_{n}[\mathrm{AlAs}]_{m}$ superlattices have been studied more extensively than any other multilayer system [5-9]. Hence, in this work, the author focusses on the influence of the interfacial effects on high- and low-angle X-ray diffraction spectra of $[\mathrm{GaAs}]_{n}[\mathrm{AlAs}]_{m}$ superlattices. The computer-simulated (using a Monte-Carlo method) spectra based on a "statistical" model of the superlattice structure are proposed in the analysis.

"Part of this work was supported by CPBP 01.08.C-1.3. 
Earlier, the author with co-workers had used succesfully the similar model prepared for studying polycrystalline metallic superlattices $[2,4,10]$. Figure 1 shows the concept of the model proposed for semiconductor superlattices.

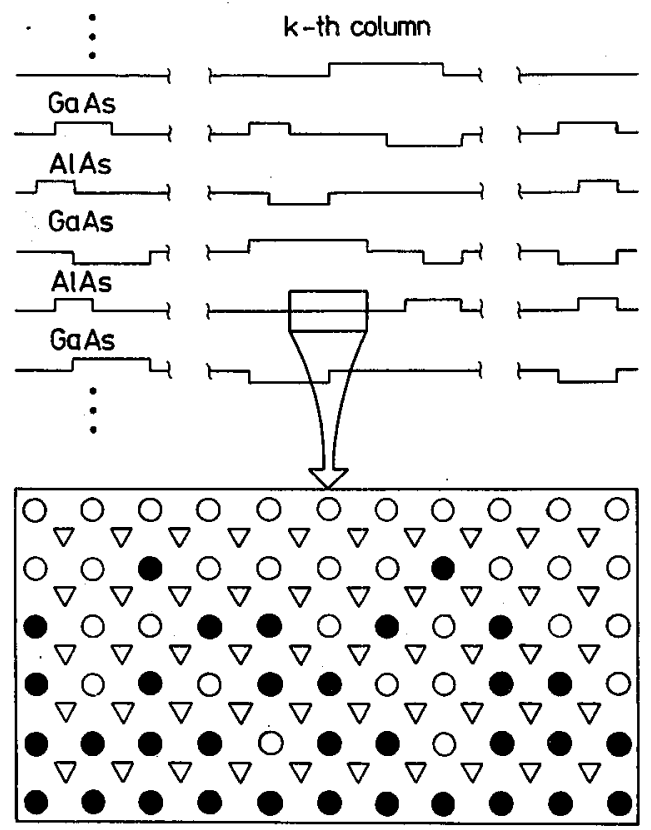

Fig. 1. Cross-sectional view of the superlattice structure grown in terraces. The interdiffused character of an interface is assumed: $-\mathrm{Ga}, \circ-\mathrm{Al}, \nabla-\mathrm{As}$ atoms.

The application of the kinematic theory of diffraction leads to the following formula in calculating the intensity $I(\theta)$ of X-rays scattered by a sample, if $\theta-2 \theta$ diffraction technique is used

$$
I(\theta)=L(\theta) \sum_{k=1}^{K}\left|\sum_{\mathrm{j}=1}^{D} P_{k j}(\theta) \exp \left(\frac{\mathrm{i} 4 \pi x_{k j} \sin \theta}{\lambda_{x}}\right)\right|^{2},
$$

where the $L(\theta)$ factor is dependent on the polarization, Lorentz and geometric factors; the $P_{k j}(\theta)$ factor [10] depends on scattering functions and Debye-Waller coefficients of atoms in the $j$-th plane of the $k$-th column; $x_{k j}$ is the coordinate of this plane in the growth direction. $D$ is the number of monolayers deposited and $\lambda_{x}$ is the $\mathrm{X}$-ray wavelength $\left(\lambda_{x}=1.54 \AA\right.$ was used in the calculation).

In order to calculate low-angle spectra it is necessary to use the dynamic theory of diffraction because in this case the absorptive dependence, refraction correction, and critical angle for total reflection must be taken into account. Parratt [11] has given a recursion formula for the intensity specularly reflected from a thin film system consisting of $N$ media which can easily be programmed using 
complex arithmetics. The reflection coefficients of X-rays at the interface between the $(j-1)$-th and $j$-th layers are given as a recursion formula

$$
R_{j-1, j}=a_{j-1}^{4}\left(R_{j, j+1}+F_{j+1, j}\right) /\left(R_{j, j+1} F_{j-1, j}+1\right),
$$

where

$$
\begin{gathered}
a_{j}=\exp \left(-\mathrm{i} \pi f_{j} d_{j} / \lambda_{x}\right), \\
F_{j-1, j}=\left(f_{j-1}-f_{j}\right) /\left(f_{j-1}+f_{j}\right), \\
f_{j}=\left(\theta^{2}-2 \delta_{j}-2 \mathrm{i} \beta_{j}\right)^{1 / 2}
\end{gathered}
$$

and where $d_{j}$ is the thickness and $1-\delta_{j}-\mathrm{i} \beta_{j}$ the refractive index of $j$-th layer. The calculation starts from a substrate which is assumed to have an infinite thickness and $R_{n, n+1}=0$ (here $n$ is the number of monolayers deposited), and it ends at the top of the sample. The ratio of reflected and incident intensities is then given by:

$$
I(\theta) / I_{o}=\left|R_{0,1}\right|^{2} .
$$

The present author wrote the computer programs based on the above-mentioned algorithms. The following parameters depending on the superlattice structure appear in the computer-modelling: the average numbers of monolayers in one superlattice unit cell, the standard deviation of the numbers of monolayers in one unit cell, the number of interdiffused monolayers at the interface. The procedures have been used to simulate low- and high-angle spectra of $[\mathrm{GaAs}]_{n}[\mathrm{AlAs}]_{m}$ superlattices. Figure 2 shows X-ray diffraction spectra calculated for the ideal $[\mathrm{GaAs}]_{15}[\mathrm{AlAs}]_{15}$ superlattice grown with (001) planes parallel to the substrate, and taking into account the statistical character of the structure. The large-angle calculations were carried out only for the superlattice structure - a substrate background was not included to the spectra because in experimental spectra the background may be easily extracted. The (a) and (d) spectra were obtained assuming the ideal structure, whereas the (b) and (e) were calculated taking into account terrace growth and fluctuations of the numbers of monolayers in the superlattice unit cell with the standard deviation $\sigma=1 \mathrm{ML}$ (monolayer). Moreover the (c) and (f) spectra were calculated assuming additionally the existence of interdiffused interfaces, each $10 \AA$ thick. Increasing of satellite peaks halfwidth, and decreasing of higher-order peaks intensities are often reported for such superlattices (e.g. in [7, 9]. However, almost ideal superlattices $[\mathrm{GaAs}]_{1}[\mathrm{AlAs}]_{1}$ and $[\mathrm{GaAs}]_{2}[\mathrm{AlAs}]_{2}$ obtention has also been reported [8]. In that case the mono- and bi-layer superlattices were synthetized by MBE using RHEED intensity oscillations technique for the deposition process control.

The model and programs proposed are useful for quick estimating the quality of experimentally obtained samples as well as for expecting the influence of interdiffusion, terrace growth and other structural parameters on X-ray diffraction spectra, and can be applied for various semiconducting superlattices. 

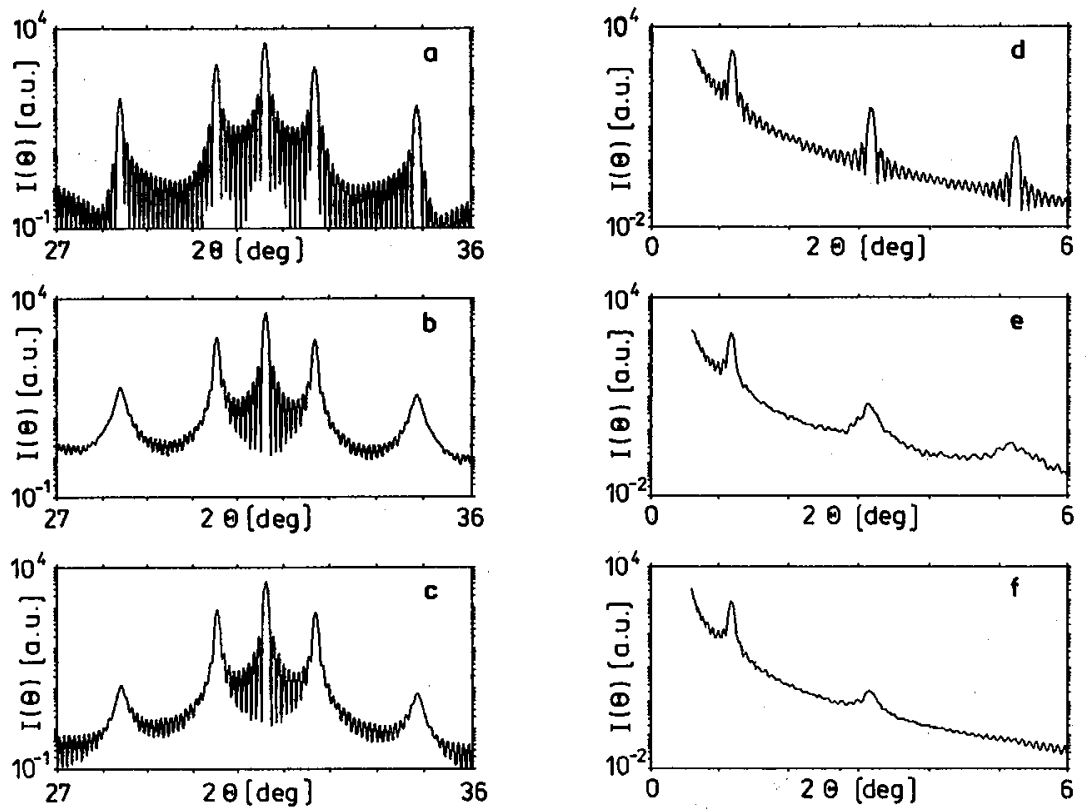

Fig. 2. High-angle and low-angle X-ray diffraction spectra of the $[\mathrm{GaAs}]_{15}[\mathrm{AlAs}]_{15}$ superlattice: (a,d) $\sigma=0 \mathrm{ML}$, Int $=0 \AA$; (b,e) $\sigma=1.0 \mathrm{ML}$, Int $=0 \AA ;(\mathrm{c}, \mathrm{f}) \sigma=1.0 \mathrm{ML}$, Int $=10 \AA$.

\section{References}

[1] L.L. Chang, B.C. Giessen, Synthetic Modulated Structures, Academic Press, New York 1985.

[2] G. Gładyszewski, Thin Solid Films 170, 99 (1989).

[3] G. Gładyszewski, Mater. Lett., in press.

[4] G. Gladyszewski, P. Mikolajczyk, Z. Mitura, M. Subotowicz, J. Phys., Condens. Malter. 1, 7795 (1989).

[5] L. Esaki, L.L. Chang, Thin Solid Films 36, 285 (1976).

[6] L.L. Chang, A. Segmüller, L. Esaki, Appl. Phys. Lett. 28, 39 (1976).

[7] R.M. Fleming, D.B. McWhan, A.C. Gossard, W. Wiegmann, R.A. Logan, J. Appl. Phys. 51, 357 (1980).

[8] N. Sano, H. Kato, M. Nakayama, S. Chika, H. Terauchi, Jpn. J. Appl. Phys. 23, L640 (1984).

[9] L. Tapfer, K. Ploog, Phys. Rev. B 33, 5565 (1986).

[10] Z. Mitura, P. Mikołajczak, J. Phys. F 18, 183 (1988).

[11] L.G. Parratt, Phys. Rev. 95, 359 (1954). 